\title{
A journey through roses and thorns: becoming a physician by learning from patients with life-threatening illnesses. A qualitative study with international medical students
}

\author{
Marina Maffoni ${ }^{1}$, Karin Olson ${ }^{2}$, Julia Hynes ${ }^{3}$, Piergiorgio Argentero ${ }^{4}$, Ilaria Setti ${ }^{4}$, Ines Giorgi ${ }^{5}$, \\ Anna Giardini ${ }^{6}$
}

${ }^{1}$ Psychology Unit, ICS Maugeri IRCCS, Institute of Montescano, Italy; ${ }^{2}$ Faculty of Nursing, Edmonton Clinic Health Academy, Edmonton, University of Alberta, Canada; ${ }^{3}$ Kent and Medway Medical School - Canterbury Christ Church University and University of Kent in collaboration, Canterbury, Kent, England; ${ }^{4}$ Department of Brain and Behavioral Sciences, University of Pavia, Italy; ${ }^{5}$ Psychology Unit, ICS Maugeri IRCCS, Institute of Pavia, Italy; ${ }^{6}$ Information Technology Department, ICS Maugeri IRCCS, Institute of Pavia, Italy

\begin{abstract}
The medical students' well-being may be threatened by various stressors associated with providing care to different kinds of
\end{abstract}

\begin{abstract}
Correspondence: Marina Maffoni, Psychology Unit, ICS Maugeri IRCCS, Institute of Montescano, Italy.

E-mail: marina.maffoni@icsmaugeri.it

Institution where the research was carried out: Department of Primary Care and Population Health, Medical School, University of Nicosia, Nicosia, Cyprus.

Acknowledgements: Heartfelt thanks to the students enrolled at the Medical School in Nicosia who have participated with sincere interest and enthusiasm. A special thanks also to Professor Peter Karayiannis who welcomed this research and to Gary Frank (Consultant Nurse) who provided us a precious external supervision.
\end{abstract}

Conflict of interest: The authors declare that they have no competing interests, and all authors confirm accuracy.

Ethical approval: The present study is part of the research project called WeDistress HELL, approved by the Ethical Committee of ICS Maugeri - Institute of Pavia (Italy), Protocol No 2211CE.

Funding: This work was partially supported by Ricerca Corrente, funding scheme of Ministry of Health, Italy.

Keywords: Medical student; life-threatening illness; risk and protective factors; qualitative research; interpretive description.

Received for publication: 2 February 2021

Accepted for publication: 21 April 2021.

${ }^{\circ}$ Copyright: the Author(s), 2021

Licensee PAGEPress, Italy

Monaldi Archives for Chest Disease 2021; 91:1800

doi: 10.4081/monaldi.2021.1800

This article is distributed under the terms of the Creative Commons Attribution Noncommercial License (by-nc 4.0) which permits any noncommercial use, distribution, and reproduction in any medium, provided the original author(s) and source are credited. patients. This study aims to explore students' clinical experiences with patients who suffer from life-threatening illnesses, focusing on potential risk and protective factors. Audio-recorded and face-toface interviews were conducted and transcribed verbatim. The "Interpretive Description" approach was used to analyse data. Overall, ten medical students with a mean age of 28 years old were interviewed. Well-being promoting factors were the following: therapeutic relationships, work-life balance, social support and communication, perception of improvement in knowledge and availability of advanced directives. Whilst factors that may reduce well-being included death exposure, managing emotions, communication difficulties, internal conflicts and disagreements, lack of knowledge and subjective concerns. These findings shed light on facets that are inherent parts of clinical experience with patients suffering from a life-threatening illness and that may turn in risk or protective factors for the medical students. Understanding the students' subjective experiences may aid in the improvement of the current educational programs, as well as in the development of tailored supportive and preventative interventions to promote well-being and professional competencies among this kind of students.

\section{Introduction}

Distress and burnout are worldwide matter of concern among healthcare professionals $[1,2]$. Medical students are affected by these issues too. Indeed, literature in this field indicates that medical students might experience anxiety, depression, suicidal ideation, distress, and burnout in response to stressful clinical experiences occurred during their internships [3-6]. About half of USA medical students complained burnout 4 and the prevalence of depressive symptoms ranged from $9.3 \%$ to almost $60 \%$ across studies with medical students [5]. Various reasons may undermine the medical students' health. Firstly, the clinical education curricula of medical schools require students to manage demanding and emotionally tough situations related to the provision of care to suffering individuals. Secondly, other factors contributing to fuel burnout among medical students include young age, lack of free time and dissatisfaction with one's educational [3]. In addition, difficulties regarding work-life balance, disproportionate workload, concerns related to career, and financial issues are also relevant self-reported stressors for medical students [7]. Conversely, some protective factors, such as 
positive emotions, seem to strengthen self-efficacy, to preserve students' well-being and to assist in reaching their professional aims [8].

The students' clinical experience becomes even more demanding when caring for individuals suffering from life-threatening illnesses. For example, frequent exposure to death and sufferingmay pave the way for experiencing negative emotions that may affect not only the students' well-being but also their performance and sense of achievement $[3,6,8]$. However, there is still scant literature focusing on the subjective experience of medical students regarding the taking care of life-threatening patients. Considering that the encounter with this kind of patients may occur inevitably somehow during the clinical tranships, the understanding of student's experiences becomes a crucial aspect that urges to be better investigated. In fact, students may feel to be unprepared to effectively manage the patient and their caregivers, as well as their own emotions [9]. Thus, it is important to further investigate the experience of medical students during their educational path in order to better tailor educational programs and effective professional curricula, trying to bridge the gaps between theory and practice [10].

Specifically, it may be useful to inquire both positive and negative facets, trying to reach a comprehensive understanding of the students' subjective experience. To this regard, qualitative research may be an effective way to reach new insights suggesting novel directions for further studies in a certain field. Therefore, we conducted a qualitative study exploring how medical students describe their clinical experience dealing with life-threatening illnesses, with the goal of identifying subjective risk and protective factors.

\section{Method}

\section{Study design}

This study is part of a broader observational multi-centre cross-sectional mixed method research project focused on wellbeing and distress experienced by healthcare professionals who provide care for individuals with life-threatening illnesses (WeDistress HELL Project).

In this exploratory qualitative study, the authors adopted the "Interpretive Descriptive" approach that helps the researchers to reach a preliminary understanding of the main patterns and themes, capturing the common and different points of view of those who experience a certain phenomenon on a daily basis [11]. Avoiding constraints and fixed schemes, the researcher is able to translate narratives into useful and coherent knowledge through an inductive analytic approach that facilitates the understanding of the phenomena under investigation and that allows the development of practical applications of the findings and the providing of suggestions for further investigations $[11,12]$. The research question guiding the present study was: "How do medical students describe their clinical experience with life-threatening patients, focusing on possible risk and protective factors?".

\section{Participants}

The participants were international medical students enrolled in a medical English-language university in southern Europe. The participation was on a voluntary basis, without any form of reinbursement. The only inclusion requirement was having had clinical experiences with patients suffering from life-threatening illnesses during their clinical placements. A satisfying proficiency level in the use of English language was guaranteed because it is a prerequisite to access to the course (minimum IELTS -International English Language Testing System - scoreof 7.0 overall).

\section{Procedure}

After the approval of Ethics Committee and general arrangements with the University, a mail explaining the project and aim of the interview were sent to all medical students, in order to present the research which was going to take place. The contact addresses of MM or JH was provided in order to ask for further details.

Through a convenience sampling technique, some medical students of the two medical programmes (4-year graduate entry and 6year direct entry), regardless of the stage of their studies, were recruited. They were provided with a link to choose a preferred slot of time for the interview. Considering the qualitative exploratory nature of the study, a small sample of informative and motivated respondents was considered satisfactory to address the research aims.

\section{Ethical considerations}

Individuals received oral and written information about the aims of the project and were askedto sign a consent form if they were interested in taking part in the study. Signing this form, students consented to the use of any written material anonymously for research purposes. A copy of the consent form was given to each participant, and they were told that they could withdraw without explanations in any moment if they wished.

\section{Data collection and analysis}

Interviews were collected in March 2019 and lasted approximately $30 \mathrm{~min}$. MM conducted face-to-face interviews, following a topic guide (Table 1). Each interview was audio-recorded and transcribed verbatim by trainee psychologists. Subsequently, MM checked for consistency between audios and transcriptions.

Table 1. Topics discussed during the interview.

1) Reasons behind the choice of studying medicine

2) (Emotional and moral) difficulties experienced during the clinical placements when providing care for a patient with a life-threatening illness

3) The most difficult aspects to manage patients suffering with a life-threatening illness

4) The helping factors in managing patients suffering with a life-threatening illness

5) Supportiveness, communication and relations in the managing of life-threatening illness

With colleagues

With superiors

6) Disagreements about treatments or caring plans for patients with life-threatening illness

With colleagues

With superiors

With patients/caregivers 
The interviews were analysed after data collection by MM and $\mathrm{KO}$, following the Interpretive Description approach [11]. The transcriptions were read to gain first impressions. After that, the transcripts were read again to ascertain the main ideas related to the research question in each interview and were compared with each other. Memos about common ideas and supporting quotes from the interviews were identified and discussed by members of the research team. Moreover, periodical briefing sessions via skype were scheduled between the research team to discuss analysis and to finalize results. Next, the results were reviewed in light of previous literature. Finally, the findings were discussed with a senior nurse caring for life-threatening patients, in order to have an external supervision. The analysis was instrumentally supported by a free qualitative analysis software program called RQDA [13].

\section{Results}

Ten students took part in this study (mean age 28.0 18.3 years; females/males 5/5; Table 2). The medical students' clinical experience may be described as a journey side-by-side with patients, with a starting point that corresponds to the decision to enrol in Medicine. During their educational path, they experience multiple interconnected factors both positive and negative, that may promote well-being as well as decrease it, fostering distress and unpleasant emotions (Figure 1).

Becoming a physician is a journey with the patient as they are busy dying, the whole thing is that it takes a lot out of you emotionally (Student 6).

\section{Starting point}

The enrolment in university to study medicine was the starting point of the educational path ofmedical students. The triggers that result in this choice were linked to interest in the human body and science, as well as to the desire, as future doctors, to help others.

I find it interesting, I find the human body fascinating, and there are so many different aspects of it that you can do, so there's always something for someone (Student 4).

I just enjoying being able to help people (Student 7).

\section{Factors that may promote wellbeing}

\section{Therapeutic relationship with the patient}

The first protective factor identified by medical students was the development of a therapeuticrelationship with the patient. This relationship opened up the possibility to empathise with patients and to think and act in an altruistic way. This approach implied an awareness of professional boundaries, too.

It is putting a line between being there for them and helping them emotionally but at the same time being like having a clear head or clear enough to take the right decision and maybe not the right decision that you think for yourself, like really taking a shared decision with the patient (Student 7).

\section{Balance between work and private life}

The capacity to maintain a balance between private life and work was also perceived as a helping factor for wellbeing. It permitted students to stay focused on the present, avoiding recursive thoughts that may otherwise consume energies and drain emotions.

You have got to compartmentalise and say that's totally that is what it is (Student 3).

I think it's about forgetting. So, when you leave work, when you close your office you have to forget about what happened in the morning and then (silence) go backhome, go out and live your life as if nothing happened and next in the morning comeback and continue (Student 8).

\section{Social support (networking, family)}

Relationships and communication with others also provided students support when they were required to witness or cope with difficult situations experienced in clinical practice. The presence of supportive people, such as family and friends, was perceived as a soothing elementand helped the student preserve their own wellbeing.

Having around the right kind of people really helps me (Student 2).

I spoke to my mom [clinician too] about it (feelings, not patient details) (Student 4).

My wife is a GP who is very, very supportive [mmm] in terms of everything (Student 10).

Table 2. Main characteristics of the interviewees.

\begin{tabular}{lcccc} 
Student & Sex & Age & Current year course & Kind of the degreecourse \\
Student 1 & Male & 20 & 1 & MD (6 years) \\
Student 2 & Female & 20 & 2 & MD (6 years) \\
\hline Student 3 & Male & 25 & 2 & MBBS (4 years) \\
Student 4 & Female & 30 & 3 & MBBS (4 years) \\
\hline Student 5 & Male & 26 & 2 & MBBS (4 years) \\
Student 6 & Female & 47 & 2 & MBBS (4 years) \\
\hline Student 7 & Female & 23 & 2 & MD (6 years) \\
Student 8 & Male & 25 & 4 & MBBS (4 years) \\
\hline Student 9 & Female & 27 & 4 & MBBS (4 years) \\
Student 10 & Male & 37 & 1 & MD (6 years)
\end{tabular}

MD, Doctor of Medicine, 6-Year Degree in Medicine; MBBS, Bachelor of Medicine, Bachelor of Surgery, 4-Year Graduate Entry Medicine Degree. 


\section{Communication with colleagues and superiors}

Another helpful element was the possibility to speak with clinical supervisors about patients and treatments. Discussing the clinical experience is supportive when facing difficulties and tough moments.

What helps me in difficult moments are colleagues and superiors, definitively, someone to talk to about these things (Student 9).

\section{Perceived improvement in knowledge and understanding}

Medical students in this study underlined the importance of perceiving improvements in termsof training, practice and knowledge in order to become a capable healthcare professional. The learning process was perceived as implicit in clinical practice and as never stopping.

I think personally... what helps me is to make me better in what I wanna do and pushes me to continue (Student 7).

I think training. Knowledge helps you the more. I do believe that the more you arefacing with this situation the more you are learning... When you are going to practical field, the practical field itself teaches you a lot of stuff, which can't be just learned from theory (Student 10).

\section{Learning from errors}

Medical students noted that being aware of errors and then accepting them as a way to learn more, helped to promote their well-being, too. This experienced interpretation refuelled the students and pushed them to do better in the future, improving their own knowledge and skills.

You should always take that case and say "Ok, what can I do better next time?" and then relive yourself of that guilt of whatever you did not do or you did wrong [silence] [...] learning more... learn more about it so the next time it does not happen (Student 9).

\section{Acceptance}

The ability to "let it go" was described as a way to accept situations, avoiding waste of time and energy. Acceptance is described as a sort of respect for the situations and for people, too.

So, respecting the situation, respecting the decision of people depending on the way that their life ends was also a massive [silence] a massive way of like getting over the issue (Student 2).

\section{Advance directive as a guide in the clinical practice}

Students reported support for the use of advance directives in clinical practice. Indeed, the presence of advance directives may reduce negative emotions when students provided care. The students perceived to have a "guidance", that is they know what to do on the base of the patient's will.

When you are rushed and when you have to make a quick decision, if you have guidance from patient regarding what they want, makes life easy for the patient and for the doctor (Student 10).

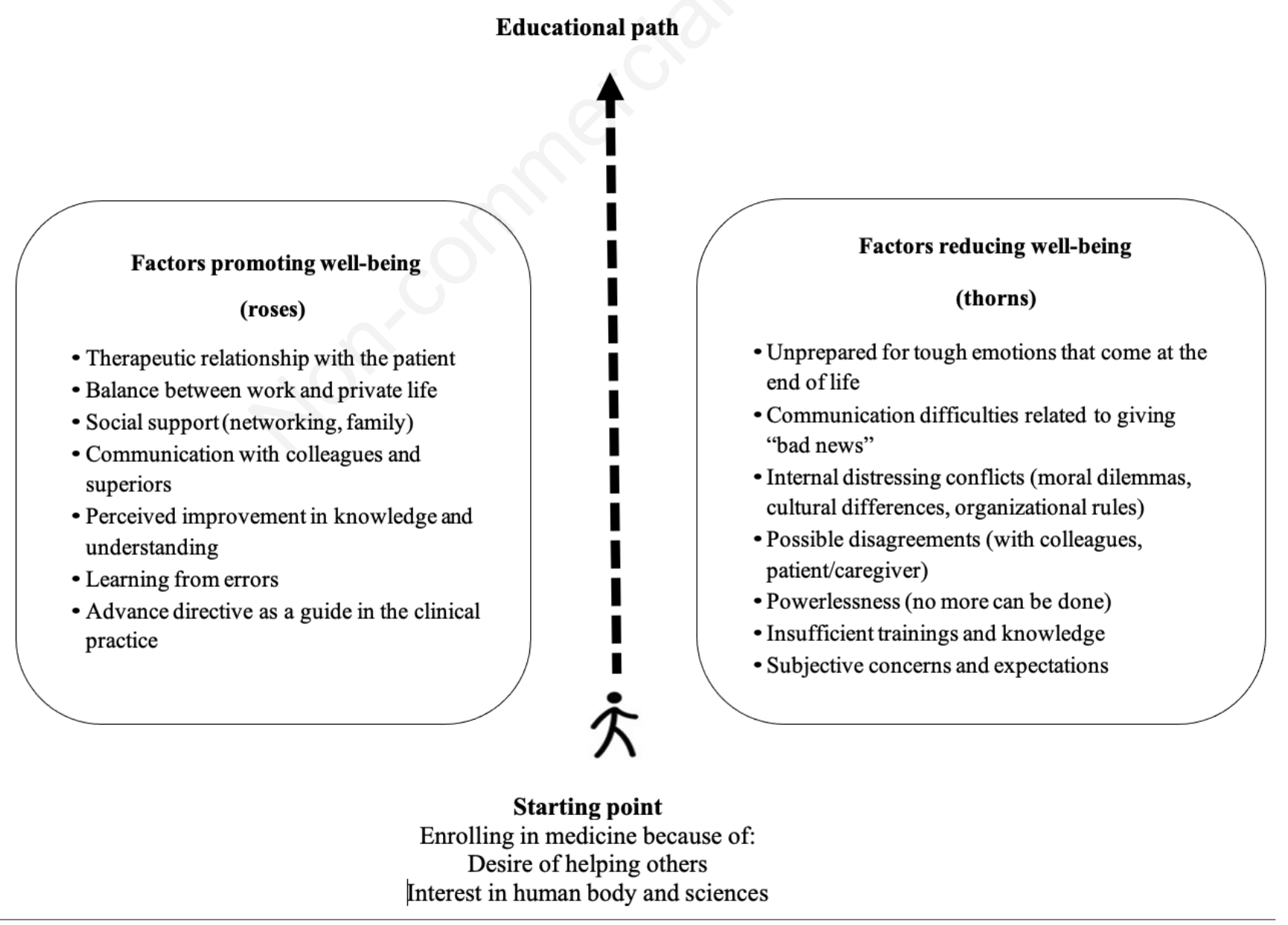

Figure 1. The scaffolding that shows how the students experience their educational path caring for life-threatening illnesses. 


\section{Factors that may potentially reduce well-being}

\section{Unprepared for tough emotions that come at end of life}

The medical students in this study noted that witnessing suffering and sudden death was "tough", "shaking", "shocking" and "sad," and they felt unprepared to manage the emotions experienced in those contexts. They were aware, of course, that they would be likely to witness these types of events, but awareness alone was not enough to overcome the emotionalresponses.

The difficult thing is... death is always difficult, no matter how prepared you thinkyou are or how much you accept it. At the end of the day, it's final and it is difficultto deal with even if you don't know the person personally [...] I was shaken... andI couldn't stop crying for a few days afterwards (Student 4).

It stacked in me because it had happened so quickly, like I was$n$ 't... when he first came in I wasn't... we all weren't expecting it [...] I also started crying ... (Student 9).

\section{Communication difficulties related to giving "bad news"}

Another issue that had the potential to reduce well-being was difficulty related to "bad news." Students noted that it was hard to find the right words and the right approach to deliver unpleasant messages. They often felt unprepared to manage this kind of communication. Situations requiring the sharing of "bad news" typically gave rise to questions from patients that were hard to answer.

I didn't know what to say and I felt bad not saying anything to reassure her, but I didn't (have) the words to say! That's what I struggled with, I didn't know what to say to reassure her (Student 4).

\section{Internally distressing conflicts (moral dilemmas, cultural differences, organizational rules)}

The students in this study experienced internal conflicts when they were required to fall intoline with difficult decisions with which they disagreed regarding patient care. Students experienced internal distress when dealing with external constraints, such as clinical protocolsand organisational rules that pushed them to act against their values.

[The student was taking care of an old woman who suddenly fell down. Although the patient sought help to stand up, the hospital procedures prescribed to ask for the help of a superior and to not move the person in order to avoid secondary damages] It was a problem where I had to follow the protocol or... to follow the situation [...]It takes, I think, a lot of courage to not do something which is naturally required from you and still follow the procedure [...] So... I think [mmm] that affected me the fact that she was crying and she was in pain (Student 10).

Cultural differences were perceived as challenging issues to accept and manage. Specifically, some procedure and practice were perceived distant and in contrast with what one is used to believe correct.

Both of them have not been informed by the medical team and often the family will say "we don't want them to know" which I think it's damaging the patient's autonomy it's, you know, stealing from the patient, really, the patient's choices and decisions [...] So it's a bit of an ethical dilemma that I sit with because yeah [...] In this particular context it's cultural but it's not ethical [...] this is not compatible with my values and culture, this is not compatible with my beliefs" or then it puts a situation where I have a conflict of, you know, in a way I'm also involuntary deceiving this patient because I'm part of the team, I'm part of the health team, even though I'm just a student... So, what do you do, you know? (Student 6).

\section{Possible disagreements (with colleagues, patient/caregiver)}

Possible disagreements with medical staff or caregivers about care provided may also be a riskfactor for well-being. Some students thought other approaches or treatment choices were more appropriate but were reluctant to voice their views.

I'm like arguing with my colleagues about how they say "No, we should give themthis treatment" and I'm like "No, they literally said they don 't want it" (Student 9).

So in that case when a patient wants to go with the treatment and I don't think it's medically viable and you want them to ehm [silence] to be better. Yes, I guess that that's the most major disagreement between the clinician and the patient (Student 3).

In most cases, the medical students in this study did not openly express disagreement with theirsuperiors because they thought it was not appropriate for individuals still in training.

I disagreed but kept it to myself because ultimately it is not my place (Student 5).

\section{Powerlessness (no more can be done)}

Powerlessness was perceived as a distressing factor, too. Students described this experience as "loss of control" and regretted they could not do more to make a difference for the patients.

The difficult thing is seeing inability, not being able to help someone that needs [mmm] not being able to do [...] So troubling, I would say, the thought of a lack ofcontrol over what could happen (Student 3).

"I wish I did more for the patient" but like yeah, I didn't know or, you know, who knows what it was best to do like there were two options and like there were pros and cons to both (Student 9).

\section{Insufficient trainings and knowledge}

The experience of not knowing enough to adequately manage demanding situations was another contributor to reduced wellbeing. Although the students recognised that they were at the outset of their medical education, they complained and felt themselves unprepared for difficult situations.

I think maybe preparation for the students would be much better before they start, like an icebreaker or something or... just an orientation, how to cope, how not to be affected (Student 8).

Not having th.e knowledge to do better, definitively, yeah, at that time you wish or specifically for that patient [...] that's the hardest part, looking back and thinking that I haven't enough information (Student 9).

\section{Subjective concerns and expectations}

Past experiences and personal characteristics of each student appeared to influence how they interpreted situations that were part of their clinical experience, potentially reducing their well-being. The practical experience gained during their placements was described as a means to adjust previous and potentially incorrect expectations.

I was dreading it for a while because I thought it would be really heartbreaking to see people that you know that are about to die [...] it [palliative care] wasn't what Iexpected, I thought there would be a lot of hysteria and a lot of sadness (Student 4).

\section{Discussion}

Ten international medical students were interviewed regarding their clinical experience with patients who were experiencing life- 
threatening illness. The findings unveiled several relevantaspects that in previous research were not often considered together.

According to the authors, the metaphor that best describes the findings of this study is a "journey through roses and thorns". This image evokes a clinical experience perceived as a travel characterised by different factors that may promote or inhibit personal well-being (Figure 1).

The journey starts with the students' enrolment at the University. The decision to choose a medical degree was linked to their interest in sciences and their desire to help others who are suffering. Since that moment, students began to think about themselves as future doctors, therefore it could be described as a time when the students start to build their professional identity as healthcare professionals $[14,15]$.

Along their educational path, the students encountered different positive factors, the so- called roses, which act as potential resources and promoters of well-being. One of the most important helping factors emerged is the ability to "adequately stay with" the patient, avoiding being too emotionally involved and professionally biased. Indeed, it was perceived as necessary to be able to control feelings in order to avoid emotional breakdown and distress [16]. Sometimes the relationship with the patient may lead to a loss of boundaries and professional identity. Consequently, the student may be unable to act with a clear mind as a healthcare professional ought. In this regard, it is importance to teach students about how to build a "therapeutic relationship" that allows the students to both empathise with the patient and to provide evidence-based care while maintaining their professional identity [17]. To this regard, the students of the present study underlined the importance to maintain a balance, that is the necessity to "compartmentalise" private life and work, staying focused in the present time and avoiding thinking about clinical experience at home. The balance between private life and professional activity was described to be a protective factor against burnout also in senior healthcare professionals $[18,19]$.

Other cornerstones described for potentially preserving students' well-being were the importance of an open communication with clinical supervisors and the possibility of receiving support from their social network (family and friends) and medical staff, too. Indeed, spending time with others and having effective communication with them may help to manage tough emotions, as well as to prevent burnout [20]. In this vein, social support may act as a buffer, moderating distress [21]. For this reason, speaking with colleagues and education supervisors may allow medical students to exchange their points of view and to share demanding situations. The same patterns have been uncovered in other healthcare students [22]. Moreover, the literature addressing healthcare professionals has underlined the importance to foster resilience and supportive environments $[2,19,23]$. This study deepened this knowledge, extending the pivotal role of social support to medical students dealing with life-threatening patients.

The interviewed students reported to perceive a constant improvement of their own knowledge and professional competencies and this perception was described as a helping factor. They appreciated the possibility to gain effective expertise through their practical clinical placements, as well as through their errors, too. Previous authors, going back as far as Aristotle and Aquinas, have confirmed the value of learning from real experience [20,24-26]. Furthermore, it is conceivable that, through this way of learning, the students may also develop their capacity to accept the course of illnesses [25]. Moreover, all interviewees showed agreement with the implementation of advance directives in clinical practice to safeguard the patients' wishes, and to guide the clinician [27]. In this regard, a previous study reported the medical students' interest in deepening their knowledge regarding advance directives during their educational curricula [28]. Moreover, a review underlined the general healthcare professionals' positive attitude toward the utilisation of advance directives [29]. Thus, the present study strengthens the relevance of advance directive also from the point of view of medical students as they can be seen as a compass to use during decision-making.

The participants to this research described some challenging risk factors, too. These elements may be described as thorns, as they are unpleasant and have the potential to decrease well-being. One of the most relevant factors potentially inhibiting well-being was the exposure to death and the management of tough and demanding emotions, such as sadness, pain, powerlessness. Students perceived not being ready when their life-threatening patients died. Some of them were overwhelmed by emotions. Similarly, the experience of nursing students who found themselves unprepared and shocked by the death of their patients has been previously described [24]. Moreover, other authors have found that the patients' death is a common experience between medical students, and supporting them to manage this occurrence is a need perceived by the learners [22]. So, it is recommended that teachers of medical students provide specific occasions to address this theme. Debriefing discussion groups may be one example of assistance, to deal with issues related to death and dying [22]. Another option might be an invitation to meet with supervisors, who could provide a safe environment for reflection by the students whose clinical exposure includes experiences with dying patients.

Another matter of concern was communication difficulties that future doctors may experience during their clinical placements. Breaking bad news concerning a diagnosis with a poor outcome was reported as a potential risk factor for well-being. In our opinion, the presence of supportive superiors is essential in this regard. Indeed, a senior clinician may help the student to move from a "cure" perspective, characterised by a never-ending search of answers, towards a "care" approach, the essence of which resides in the ability to provide emphatic listening. In this way, it is possible to foster the shift from a cure to a care orientation approach in medicine [30]. Indeed, the interviewed students were troubled as they perceived themselves lacking in communications and interpersonal skills in this regard. Literature on senior healthcare professionals has already demonstrated this aspect as a critical issue with which individuals face [24,28]. Although a previous research reported that medical students consider communication skills something natural and linked to expertise and practice [28], in the present studies the students welcomed and fostered communication trainings during their educational path, in particular regarding how to manage the breaking of bad news. In a similar way, disagreement and difficulty in communications with colleagues and superiors may also become a matter of distress for students, as already reported by other professionals $[2,19]$.

Moreover, the students interviewed reported difficulties in following practices and rulesthat are not in line with their own values [16,31]. This internal conflict, leading to a decline in well-being, is known in literature as moral distress and it affects many healthcare professionals, particularly so when dealing with bioethical and moral issues $[1,2,32]$. In this regard, senior clinicians may support the medical students towards the understanding and the adoption of a taking care approach in accordance with local habits and customs, to adequately take care of their patients and caregivers.

Lastly, knowledge, subjective concerns and expectations may affect the students' well-being during their practical clinical placements. Indeed, the students interviewed reported difficulties linked to the lack of sufficient know-how and prior negative expectations regarding taking care of patients with life-threatening illnesses. However, improving knowledge and practice with regards to this 
kind of patients changed expectations and ameliorated their clinical experiences [24]. Practising in real contexts may allow students to experience what it really means to work with life-threatening patients in a scholarly manner, and thus become more aware of the importance of the never-ending learning processes, too [25].

\section{Practical implications}

Although further investigations are needed, these findings may highlight the following points as suggestions that could be used to develop tailored educational curricula:

- To promote trainings focused on how to build a therapeutic relationship with the patients and how to adopt an effective communication (e.g., how to break bad news) both with the patient and with their caregivers;

- To increase technical knowledge and practical training enhancing and anticipating the clinical placements with life-threatening illnesses;

- To foster occasions and facilities where the students can reflect on their clinical experienceand be supported by superiors.

\section{Strengths and limits}

The main strength of this research resides in the focus on manifold risk and protective factors in the same investigation, providing a comprehensive understanding of students' experience in touch with a specific kind of patients, that is individuals suffering from life-threatening illnesses. Moreover, another strength point was the adoption of an approach useful to develop a practice-relevant knowledge valuable to improve educational programs for medical students, bridging the gap between theory and clinical practice. Indeed, using the Interpretive Description approach, the research team was able to detect protective and risk factors characterizing the experience of medical students who dealt with life-threatening patients $[11,12]$. In this way, medical students' narratives turn into powerful instruments to improve their training and educational path. Moreover, the flexible but rigorous method enabled the researchers to conduct a coherent preliminary inquiry that may provide a baseline for further studies on the same topic [12].

Nevertheless, some limits have to be underlined. Firstly, the sample size did not allow safe generalization. However, this study has to be intended as a preliminary investigation. Thus, these findings provided only suggestions for further studies and fuel deeper reflections on actual educational programs. Secondly, the not considered differences in the length of clinical placements in touch with life-threatening patients may be a bias of the findings. Finally, it hasto be underlined that the multi-nationality of the sample may be both a value and a limitation.

However, the size of the sample and the nature of the study did not allow to do further reflections about possible cultural differences or common traits.

\section{Conclusions}

In this exploratory study, the authors attempted to shed light on students' clinical experience with life-threatening illnesses, highlighting the critical issues to be managed, as well as the helping factors to be exploited in support of interventions and educational practice. Indeed, to safeguard the well-being and to respond to needs of medical students of today is imperative, in order to preserve the well-being of our physicians of tomorrow [33,34].

\section{References}

1. Hynes J, Maffoni M, Argentero P, et al. Palliative medicine physicians: doomed to burn? BMJ Support Palliat Care 2019;9:45-6.

2. Maffoni M, Argentero P, Giorgi I, et al. Healthcare professionals' moral distress in adult palliative care: a systematic review. BMJ Support Palliat Care 2019;9:245-54.

3. Costa EF, Santos SA, Santos AT, et al. Burnout Syndrome and associated factors among medical students: a cross-sectional study. Clinics 2012;67:573-80.

4. Dyrbye LN, Thomas MR, Massie FS, et al. Burnout and suicidal ideation among US medical students. Ann Intern Med 2008;149:334-41.

5. Rotenstein LS, Ramos MA, Torre M, et al. Prevalence of depression, depressive symptoms, and suicidal ideation among medical students: A systematic review and meta-analysis. JAMA 2016;316:2214-36.

6. Thurn T, Anneser J. Medical students' experiences of moral distress in end-of-life care. J Palliat Med 2020;23:116-120.

7. Hill MR, Goicochea S, Merlo LJ. In their own words: stressors facing medical students in the millennial generation. Med Educ Online 2018;23:1530558.

8. Burr J, Beck Dallaghan GL. The relationship of emotions and burnout to medical students' academic performance. Teach Learn Med 2019;2019:1-8.

9. Boland JW, Barclay S, Gibbins J. Twelve tips for developing palliative care teaching in an undergraduate curriculum for medical students. Med Teach 2019;41:1359-65.

10. Kitto S. "What is an Educational Problem?" Revisited. J Contin Educ Health Prof 2019;39:223-4.

11. Thorne S. Interpretive description: Qualitative research for applied practice. London: Routledge; 2016.

12. Hunt MR. Strengths and challenges in the use of interpretive description: reflections arising from a study of the moral experience of health professionals in humanitarian work. Qual Health Res 2009;19:1284-92.

13. Ronggui H. RQDA: R-based Qualitative Data Analysis. R package version 0.2-8; 2016. Retrieved May 2019.

14. Hatem DS, Halpin T. Becoming doctors: Examining student narratives to understand the process of professional identity formation within a learning community. J Med Educ Curric Dev 2019;6:2382120519834546.

15. Wilson I, Cowin LS, Johnson M, et al. Professional identity in medical students: pedagogical challenges to medical education. Teach Learn Med 2013;25:369-73.

16. Sasso L, Bagnasco A, Bianchi M, et al. Moral distress in undergraduate nursing students: A systematic review. Nurs Ethics 2016;23:523-34.

17. Percy M, Richardson C. Introducing nursing practice to student nurses: How can we promote care compassion and empathy. Nurse Educ Pract 2018;29:200-5.

18. Shanafelt TD, Hasan O, Dyrbye LN, et al. Changes in burnout and satisfaction with work- life balance in physicians and the general US working population between 2011 and 2014. Mayo Clin Proc 2015;90:1600-13. 
19. Maffoni M, Argentero P, Giorgi I, et al. Underneath the white coat: Risk and protective factors for palliative care providers in their daily work. J Hosp Palliat Nurs 2020;22:108- 14.

20. Gadoud A, Adcock Y, Jones L, et al. "It's not all doom and gloom": perceptions of medical students talking to hospice patients. J Palliat Med 2013;16:1125-9.

21. Uchino BN, Carlisle M, Birmingham W, et al. Social support and the reactivity hypothesis: conceptual issues in examining the efficacy of received support during acute psychological stress. Biol Psychol 2011;86:137-42.

22. Gallagher O, Saunders R, Tambree K, et al. Nursing student experiences of death and dying during a palliative care clinical placement: Teaching and learning implications. In Transformative, innovative and engaging. Proceedings of the 23rd Annual Teaching Learning Forum, 30-31 January 2014. Perth: The University of Western Australia. Available from: http://ctl.curtin.edu.au/professional_development/conferences/tlf/tlf2014/refereed/gallagh er.pdf

23. Zanatta F, Maffoni M, Giardini A. Resilience in palliative healthcare professionals: a systematic review. Support Care Cancer 2020;28:971-8.

24. Ranse K, Ranse J, Pelkowitz M. Third-year nursing students' lived experience of caring for the dying: a hermeneutic phenomenological approach. Contemp Nurse 2018;54:160-70.

25. Head BA, Earnshaw LA, Greenberg RB, et al. "I will never forget": what we learned from medical student reflections on a palliative care experience. J Palliat Med 2012;15:535-41.

26. Hynes J. I want you to pretend to be sincere. Med Educ 2016;50:1219-23.
27. Maffoni M, Argentero P, Giorgi I, et al. Healthcare professionals' perceptions about the Italian law on advance directives. Nurs Ethics 2020;3:796-808.

28. Wittenberg-Lyles EM, Goldsmith J, Ragan SL, et al. Medical students' views and ideas about palliative care communicatio training. Am J Hosp Palliat Care 2010;27:38-49.

29. Coleman AM. Physician attitudes toward advanced directives: a literature review of variables impacting on physicians attitude toward advance directives. Am J Hosp Palliat Care 2013;30:696-706.

30. De Valck C, Bensing J, Bruynooghe R, et al. Cure-oriented versus care-oriented attitudes in medicine. Patient Educ Couns 2001;45:119126.

31. Bordignon SS, Lunardi VL, Barlem ELD, et al. Moral distress in undergraduate nursing students. Nurs Ethics 2019;23: 523-34.

32. Lamiani G, Borghi L, Argentero P. When healthcare professionals cannot do the right thing: A systematic review of moral distress and its correlates. J Health Psychol 2017;22:51-67.

33. Maffoni M, Sommovigo V, Giardini A, et al. Dealing with ethical issues in rehabilitation medicine: The relationship between managerial support and emotional exhaustion is mediated by moral distress and enhanced by positive affectivity and resilience. J Nurs Manag 2020;285:1114-25.

34. Maffoni M, Sommovigo V, Giardini A, et al. Well-being and professional efficacy among health care professionals: The role of resilience through the mediation of ethical vision of patient care and the moderation of managerial support. Eval Health Prof 2021; 1632787211042660. Online ahead of print. 